\title{
Simulation of the neutron rate in ASDEX Upgrade
}

\section{H-mode discharges}

\author{
G. Tardini †, C. Höhbauer, R. Fischer, R. Neu, and the ASDEX \\ Upgrade Team
}

Max-Planck-Institut für Plasmaphysik, EURATOM Association, Boltzmannstraße 2, 85748 Garching, Germany

\begin{abstract}
The neutron rate is a direct indicator of the fusion performance in tokamaks. However, the accuracy of the measurement is limited by the wide range, covering several orders of magnitude, and by the delicate absolute calibration procedure. Moreover, a mere neutron counter does not distinguish between thermonuclear, beam-plasma and beam-beam fusion reactions. In this work we aim to use Monte Carlo simulations of the NBI deposition to improve our physics understanding of the correlation of the neutron yield with global plasma parameters. The modelling shows the beam-plasma reactions to be the main contribution to the neutron rate for $P_{N B I}>5 \mathrm{MW}$. The comparison between experimental data and measurements allows to identify systematic calibration factors for different calibration phases over several years of acquisition. The quality of the kinetic profile measurements is shown to play an important role in reducing the scatter of the simulated neutron rate around the measured value. The sensitivity to $\mathrm{Z}_{\text {eff }}$ and to possible fast ion
\end{abstract}

‡ Corresponding author: git@ipp.mpg.de 
diffusion is investigated. Using an existing formula for the density dependence of $\mathrm{Z}_{\text {eff }}$ the agreement between theoretical prediction and experimental measurements is considerably improved. Finally we derive a scaling law for the measured neutron rate in ASDEX Upgrade H-mode discharges depending on global parameters, in excellent agreement with a simple physics derivation for beam-plasma neutrons.

Keywords: Neutron production 


\section{Introduction}

The neutron production is a direct, inexpensive measurement of the fusion performance in a fusion device like a tokamak. However its intepretation is not straightforward due to the several parameters it depends on. In particular, in case of strong deuterium Neutral Beam Injection (NBI) heating the neutron rate does not reflect the energy confinement, becoming rather uncorrelated from the triple product $n T_{i} \tau$, the figure of merit of the fusion gain $\mathrm{Q} /(\mathrm{Q}+5)$ [1]. The dominant players in that case are the NBI ion energy and their density, meaning that the slowing down time plays a key role. In addition, beam deuterons can enter a fusion reaction among themselves. As a consequence, to interpret the measured neutron rate in NBI heated discharges (such as most H-mode plasmas) one needs well diagnosed kinetic profiles and an accurate model for the NBI deposition and the kinematics of the beam fast ions.

Moreover, a higher impurity concentration reduces the neutron rate at a given electron density. Further physics effects such as anomalous transport of fast ions, which could be caused by microscale turbulence or by MHD activity, would decrease the fast ion population and therefore also the neutron production. While $\mathrm{Z}_{\text {eff }}$ is usually measured with large uncertainty, anomalous transport of fast ions cannot be measured directly and so far there is no common evidence of its quantitative extent [2] [3] [4]. Assuming neoclassical fast ion transport, the neutron rate can be used as a consistency check of the kinetic and impurity profiles [5] [6][7]. Conversely, in case of a reliable assumption for the impurity concentration, the neutron rate can be used to estimate the required 
diffusion coefficient of the fast ions.

In this paper we focus on ASDEX Upgrade conventional and improved H-modes, excluding ICRH phases where the study of synergy effects with NBI is on-going [9]. Taking experimental kinetic profiles we model the NBI deposition and the fast ion orbits with the MonteCarlo package NUBEAM within the TRANSP code [10]. A similar method has been used recently to model the neutron count of a neutron camera at MAST [8]. A few assumptions on the impurity concentration are compared, and a sensitivity study with respect to $\mathrm{Z}_{\text {eff }}$ and the anomalous fast ion diffusion coefficient $D_{f i}$ are presented in Section 5. Systematic calibration effects over several campaigns are evaluated on the basis of the modelling. The importance of good quality electron density measurement is highlighted. A new set of simulations is discussed in Section 6, where $\mathrm{Z}_{\text {eff }}$ is not kept constant anymore, but it is rather determined by an empirical formula for ASDEX Upgrade H-mode discharges [11]. In Section 7, the re-calibrated experimental data are fitted with a power regression with respect to the most relevant plasma paramters. This regression is explained in terms of the underlying physics, exhibiting a good agreement with the derived formula. Finally, conclusions are presented in Section 8.

\section{Database description and diagnostics}

A database of 144 ASDEX Upgrade H-mode discharges is selected, all performed between 1998 and 2008. During this time, the neutron rate counters have been calibrated 
10 times; each discharge has been labelled according to the calibration phase, in order to account for possible systematic effects. Moreover, the ASDEX Upgrade wall was coated step-wise with tungsten, starting in 1998 and finishing in 2006 [12]. The quality of the density profile measurement improved over the years, especially since the Integrated Data Analysis is available [13], based on the experimental measurements from interferometry and lithium beam diagnostics.

The discharges are chosen to be representative, that is general enough, without any particular restriction on the NBI geometry: there is both perpendicular and tangential injection. No specific constraints are applied concerning MHD activity, except that the time intervals have to be quasi-stationary. Moreover, the following selection criteria are imposed:

- only discharges with significant neutron rates are considered, the threshold being chosen to be $10^{14} \mathrm{~s}^{-1}$

- only quasi-stationary phases are selected, with respect to plasma energy, plasma density and neutron rate;

- ICRH heated phases are excluded, because synergy effects between NBI deposition and radio-frequency absorption are not yet fully understood [9] and anyway not included in the MonteCarlo ion distribution function within TRANSP.

For two discharges, \# 11207 and \# 11208, two time intervals with different NBI power and significantly different neutron rates are picked, for a total of 146 time points. As some of the input profiles necessary for the TRANSP simulation are missing or not 


\begin{tabular}{|c|c|c|c|}
\hline Parameter & Unit & Minimum value & Maximum value \\
\hline$I_{p l}$ & $\mathrm{MA}$ & 0.8 & 1.2 \\
$P_{N B I}$ & $\mathrm{MW}$ & 4.6 & 13.9 \\
$H_{I P B 98(y, 2)}$ & & 0.83 & 1.66 \\
$\bar{n}_{e}$ & $10^{19} \mathrm{~m}^{-3}$ & 3.3 & 11.1 \\
$T_{e}(0)$ & $\mathrm{keV}$ & 1.3 & 5.8 \\
$T_{i}(0)$ & $\mathrm{keV}$ & 1.9 & 11.9 \\
\hline
\end{tabular}

Table 1. Ranges of the main plasma and enginieering parameters of the considered discharge database.

validated, a subset of 108 experimental time points constitutes the modelling database. The electron density profiles are taken, whenever possible, from the Integrated Data Analysis, a method for combining and optimising the information contained in the interferometer and lithium beam diagnostics [13]. When IDA is not available, the interferometry measurements are Abel-inverted. The electron temperature is measured with the electron cyclotron emission. Ion temperature and toroidal rotation velocity profiles are taken from charge exchange recombination spectroscopy. All profiles have been smoothed by a cubic spline before being fed into the TRANSP code.

The ranges of the main plasma and engineering parameters are summarised in Table 1. There $I_{p l}$ is the plasma current, $H_{I P B 98(y, 2)}$ the confinement improvement factor according to the IPB98 $(\mathrm{y}, 2)$ scaling law $[14], \bar{n}_{e}$ is the line-averaged electron density, $T_{e}(0)$ and $T_{i}(0)$ are the central electron and ion temeperatures, respectively. The neutron 
yield is measured with an array of 5 detectors [15], covering a range between $10^{9}$ and $10^{16}$ neutrons/second. A BF3 detector is used for the absolute calibration, while the others are cross-calibrated in the overlapping ranges. Since the performance of the detectors changes over the years, as well as the hardware between the tokamak chamber and the detector location, calibrations are performed roughly every second year. Details concerning the detectors and the calibrations procedure can be found in [15].

\section{Modelling assumptions}

In a tokamak plasma, unless tritium is injected, the fusion reactions are almost exclusively D-D reactions:

$$
\begin{gathered}
D+D \rightarrow{ }_{2}^{3} \mathrm{He}[0.82 \mathrm{MeV}]+n[2.45 \mathrm{MeV}] \\
D+D \rightarrow T[1.01 \mathrm{MeV}]+p+[3.02 \mathrm{MeV}]
\end{gathered}
$$

Both reactions are equally likely, but only the first one produces neutrons. Additionally there are some burn-up D-T reactions, but their rate is negligible compared to the amount of D-D reactions [16][17]. Due to the high reactivity for deuterium energy above $50 \mathrm{keV}$, NBI injected deuterons can dominate the fusion reactions despite their lower density compared to the thermal $\mathrm{D}$ in the plasma. Moreover, the beam deuterons can undergo fusion reactions among themselves, although this is expected to be a minor contribution, as the fast ion density $n_{f i}$ counts quadratically, while the deuterium energy is roughly the same as for beam-plasma fusion. In the ASDEX upgrade plasma discharges up to 2008 these three terms could not be detected 
separately, as no information on the neutron energy was recorded. The recently installed neutron spectrometry diagnostics [16][17] enables to deliver complementary experimental information, as soon as the characterization of the detector will enable to deconvolve the measured pulse height spectra. There can be, however, a signature of which component is dominant if one looks at how the neutron rate scales with a certain set of plasma parameters. Substituting the approximation $\tau_{E} \propto T_{e}^{1.5} n_{e}^{-1}$ into the 0D derivation presented in [18], equation 14, yields:

$$
\begin{aligned}
& S_{t h} \propto n_{D}^{2} T_{i}^{\alpha}, \alpha \approx 6.27 T_{D}[k e V]^{-1 / 3}-\frac{2}{3} \\
& S_{b t} \propto T_{e}^{1.5} \frac{n_{D}}{n_{E}} P_{N B I} \\
& S_{b b} \propto P_{N B I}^{2} E_{i n j}^{-2} T_{e}^{3} n_{e}^{-2}
\end{aligned}
$$

where $E_{i n j}$ is the beam injection energy, $n_{D}$ is the deuterium density and $S$ represents a neutron rate: the subscripts $t h, b t$ and ${ }_{b b}$ refer to the thermonuclear, beam-target and beam-beam neutron rates, respectively. In the TRANSP output the three contributions to the neutron production can be separated. In fact, the code describes the fast ions orbits, starting from their ionisation location and including collisions with the background plasma, which slows down the suprathermal ions. At every time step a fast ion distribution function is determined on a $2 \mathrm{D}$ spatial grid. Its convolution with the fusion cross-sections from [19] and a maxwellian distribution yields the beam-plasma fusion rate, whereas a self-convolution returns the beam-beam reaction rate. For the beam ionisation the Janev model [20][21] is used. A dedicated study has shown that using different beam stopping cross sections has a negligible effect on the NBI deposition 
profile for ASDEX Upgrade H-mode discharges [21]. The effect becomes slightly more sizeable only for very high plasma density, $n_{e} \approx 10^{20} \mathrm{~m}^{-3}$, and only for off-axis NBI. The effect of a different model for the beam stopping cross-sections on the beam-target neutron rate is even more negligible, because the NBI deposition profile is always broad and the fast ions density is even broader due to neoclassical transport.

The MHD activity, which can be present in some of the discharges, is not modelled, therefore the direct effect of MHD on fast ions is a possible source of uncertainty in our study. The indirect effects of MHD affecting the neutron rate via the kinetic profiles, instead, are of course retained in the modelling, since the kinetic profiles are not simulated but rather imposed from the experimental measurements.

\section{Modelling results}

The profile database is then used as input for TRANSP/NUBEAM simulations. Since $\mathrm{Z}_{\text {eff }}$ profiles are not routinely measured and, when available, they are diagnosed only with significant uncertanties, we have first assumed a rule-of-thumb value $\mathrm{Z}_{\text {eff }}=1.8$ constant in time and space, with carbon as plasma impurity in the modelling. At this stage, zero diffusion has been assumed for suprathermal ions. The modelled neutron rate is compared to the experimental one in Fig. 1, in which different colours refer to different calibration phases. As it can be seen, there are some systematic effects linked to the different calibrations, but also a significant scattering within each calibration phase. To quantify the systematic deviation due to calibration the data are fitted with a linear 


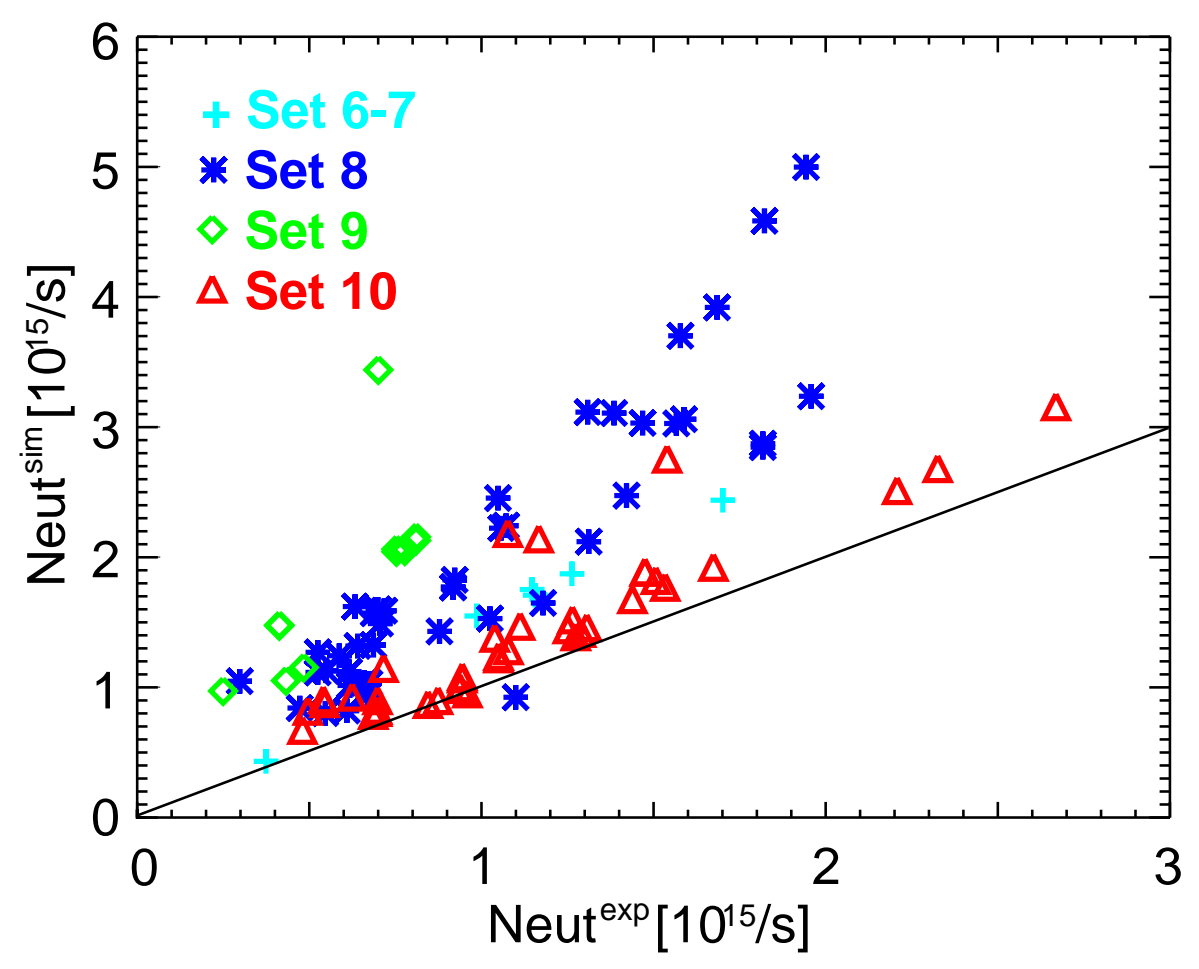

Figure 1. Modelled neutron rate versus the experimental measurement, assuming $\mathrm{Z}_{e f f}=1.8$ constant in time and space. Different colours and symbols are associated with different calibration phases.

regression forced through the origin. For a quantitative evaluation of the scattering within a given calibration set, we use the correlation coefficient between the ratio of simulated to measured neutron rate and the identity straight line. This procedure provides a tool to judge the quality of the input for the modelling, both the assumed and the measured one.

Figure 1 shows that the experimental neutron rate is systematically overpredicted by the simulations. Moreover, there is a clear trend to an even stronger overestimate at low electron density, as illustrated in Fig. 2. In the next sections we investigate the possible origin of these deviations. 


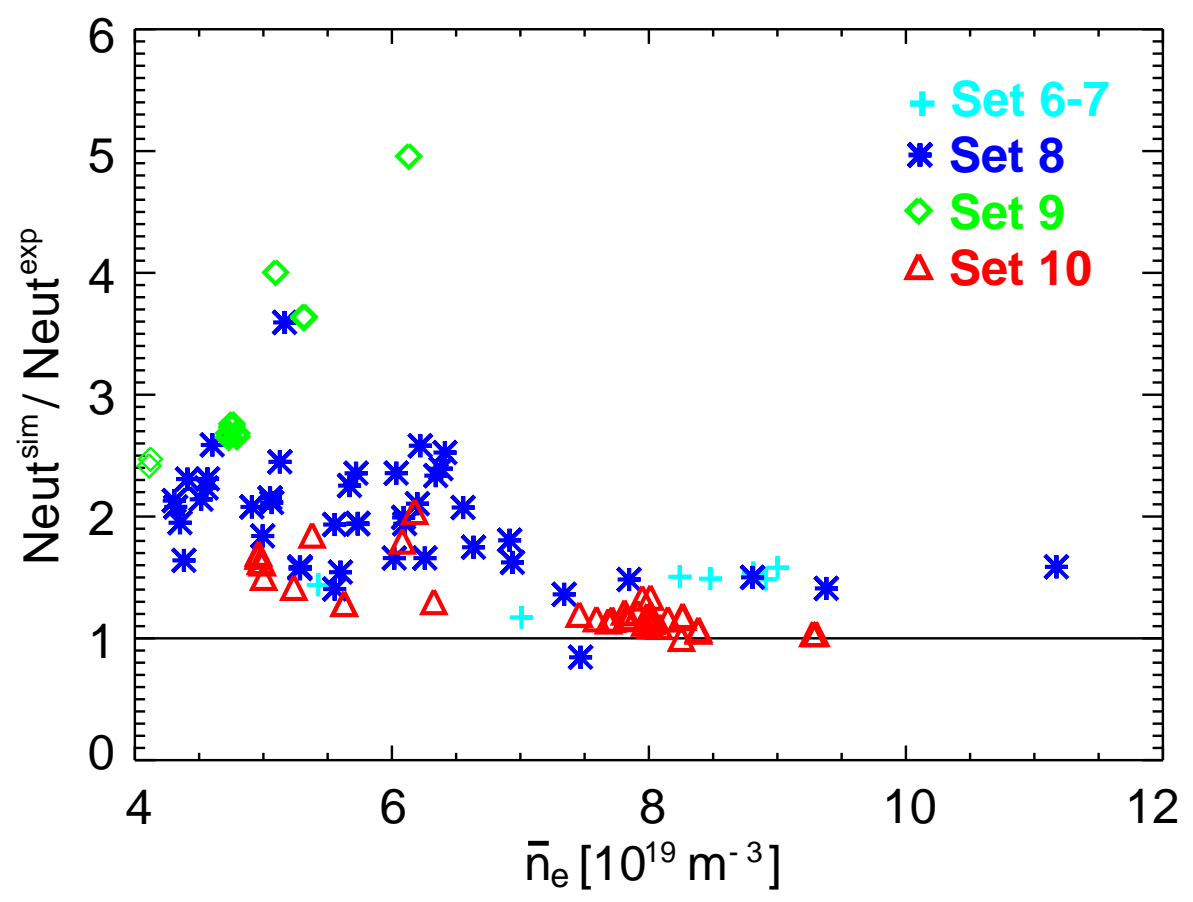

Figure 2. Ratio between modelled and experimental neutron rate versus $\bar{n}_{e}$, assuming $\mathrm{Z}_{\text {eff }}=1.8$ constant in time and space. Colors and symbols like in Fig. 1.

\section{Sensitivity studies}

\subsection{Sensitivity to the diffusion coefficient}

Fast ions are transported across the magnetic surfaces due to neoclassical transport.

This is implicitly included in the NUBEAM package, where single collision processes of suprathermal ions are computed. Furthermore, evidence for anomalous transport of fast ions has been found both on the experimental side, such as in D-alpha measurements [22] and NBI current drive studies [2] and in theory [23]. In some cases a finite diffusion coefficient of fast ions is invoked to ensure an accurate matching of the experimental neutron emission [7][24]. In most tokamaks, in fact, the Fokker-Planck simulations of the NBI lead to a sizeable overestimate of the neutron rate, as documented for TFTR 
NBI-heated plasmas [25]. It is therefore important to assess quantitatively the size of a possible diffusion coefficient in order to have a sizeable effect on the modelled neutron rate. We use the same subset of our database employed already for the $\mathrm{Z}_{\text {eff }}$ scan. The diffusion coefficient $\mathrm{D}_{f i}$ is scanned between 0 and $4 \mathrm{~m}^{2} / \mathrm{s}$. The outcome is displayed in Fig. 3. With increasing diffusion coefficient the calculated neutron rate decreases,

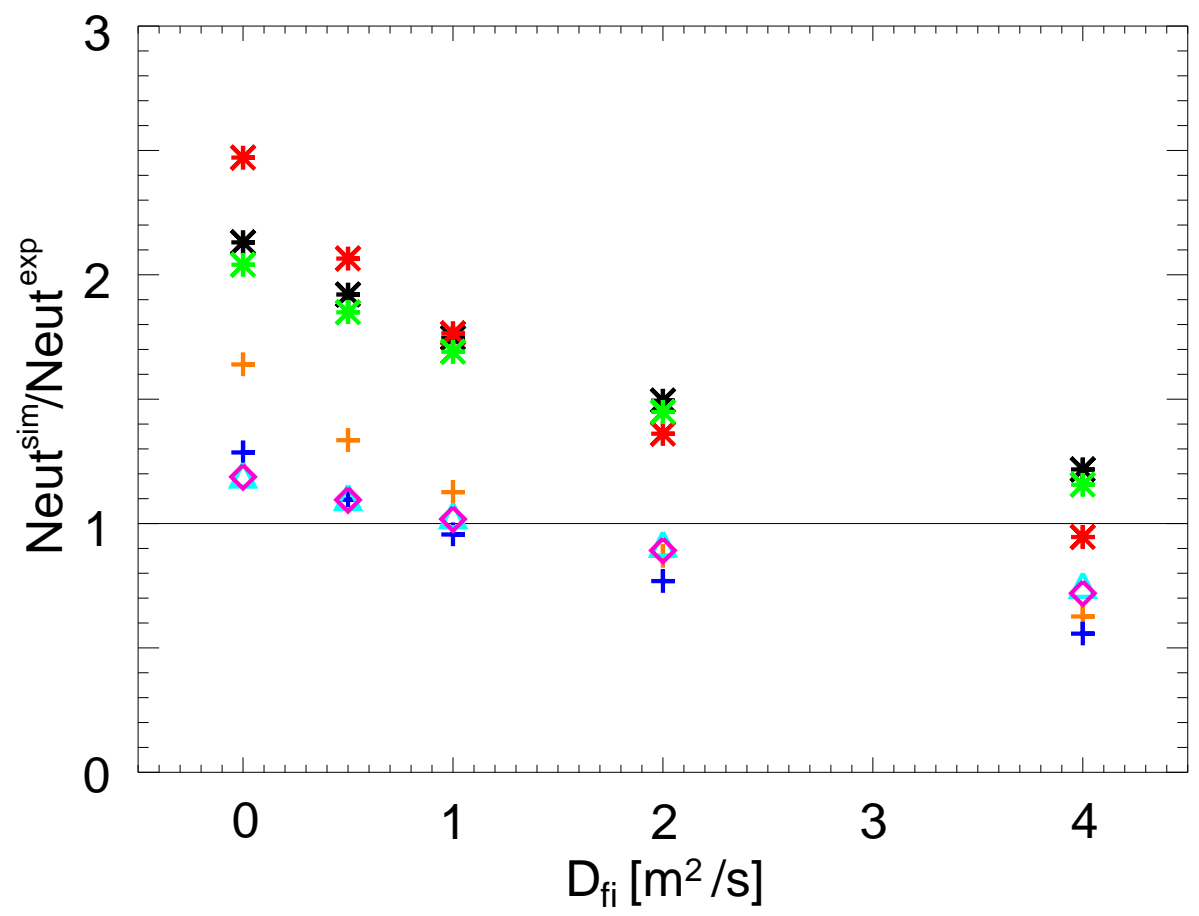

Figure 3. Sensitivity of the neutron rate to $\mathrm{D}_{f i}$ assuming $\mathrm{Z}_{\text {eff }}=1.8$ constant in time and space. Discharges with lower $n_{e}$ (star symbols; colors black, red, green) are more affected by a $\mathrm{D}_{f i}$ increase.

as expected, because more fast ions are transported out of the plasma. As expected, discharges with lower density and hence higher slowing down time show the largest reduction in the neutron rate, thus compensating the trend of Fig 2. 


\subsection{Sensitivity to $Z_{\text {eff }}$}

The results in Fig. 2 suggest that there is possibly a systematic error on $Z_{\text {eff }}$, which can indeed be expected to be higher at lower plasma density. Therefore, a sensitivity study is performed, in order to assess whether realistic $\mathrm{Z}_{\text {eff }}$ values can produce significant effects on the neutron rate under the experimental conditions. A subset of 8 discharges is selected, covering a density range between 5 and $9 \cdot 10^{19} \mathrm{~m}^{-3}$. For each discharge $\mathrm{Z}_{\text {eff }}$ was varied from 1.5 to 3 , always keeping carbon as main impurity. The results are shown in Fig. 4. The decrease of the neutron rate is obviously linear with the

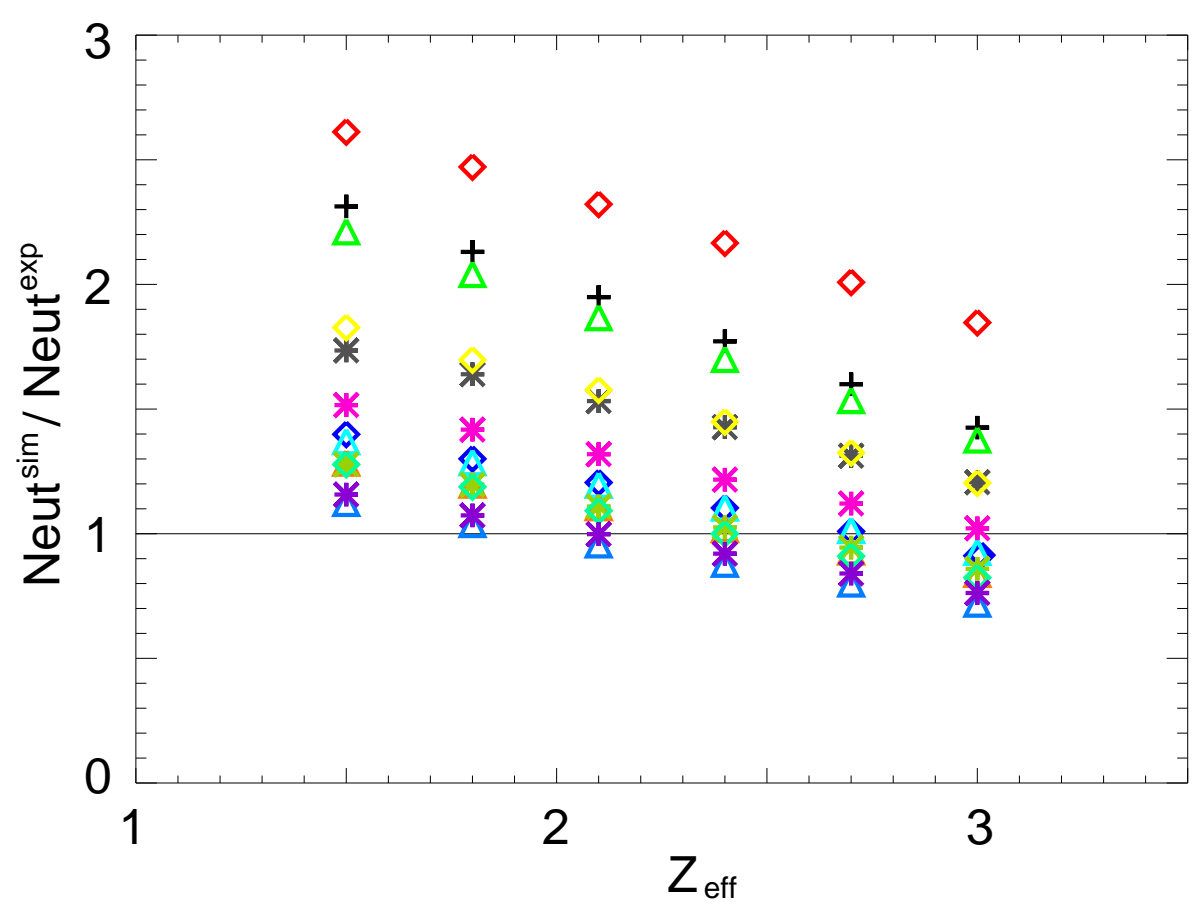

Figure 4. $\mathrm{Z}_{\text {eff }}$ scan for a subset of discharges in a density range $5-9 \cdot 10^{19} \mathrm{~m}^{-3}$.

$\mathrm{Z}_{\text {eff }}$ increase, suggesting that beam-plasma fusion is the dominant contribution. The verification of this hypothesis is discussed in Section 6 . 


\section{Simulations with density dependent $\mathrm{Z}_{e f f}$}

A new set of simulations is performed, with a more founded assumption for the $\mathrm{Z}_{\text {eff }}$ profile, still constant in space, but depending on the line averaged electron density according to the empirical formula derived for ASDEX Upgrade H-mode discharges:

$$
Z_{\text {eff }}=\min \left(1+\frac{11.2}{\bar{n}_{e}-0.74}, 4.5\right)
$$

published in [11]. In Fig. 5 the new simulation results are plotted against the experimental neutron rate (same colour code). The figure shows a systematic

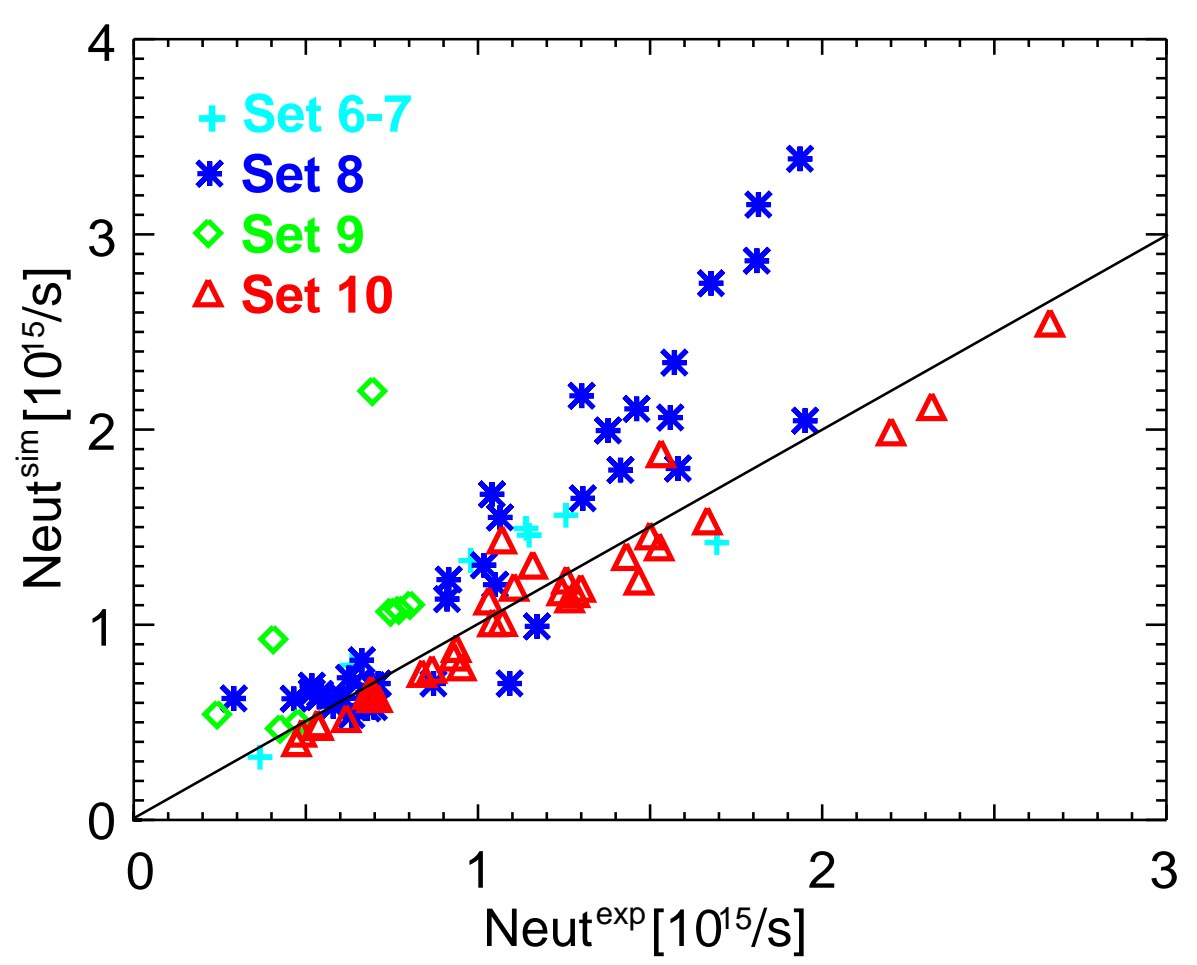

Figure 5. Modelled neutron rate versus the experimental measurement, assuming a density dependent $\mathrm{Z}_{\text {eff }}$ value. Colors and symbols like in figure 1 .

improvement, as the higher $\mathrm{Z}_{\text {eff }}$ value reduces the predicted neutron rate, therefore closer to the measured one. Furthermore, the scattering is reduced within each 
calibration phase, so the assumption for $\mathrm{Z}_{\text {eff }}$ can be regarded as more accurate over the whole database. Moreover, the systematic trend with the plasma density observed in Fig. 2 is removed, as Fig. 6 illustrates. The fit coefficient, inverse of the calibration

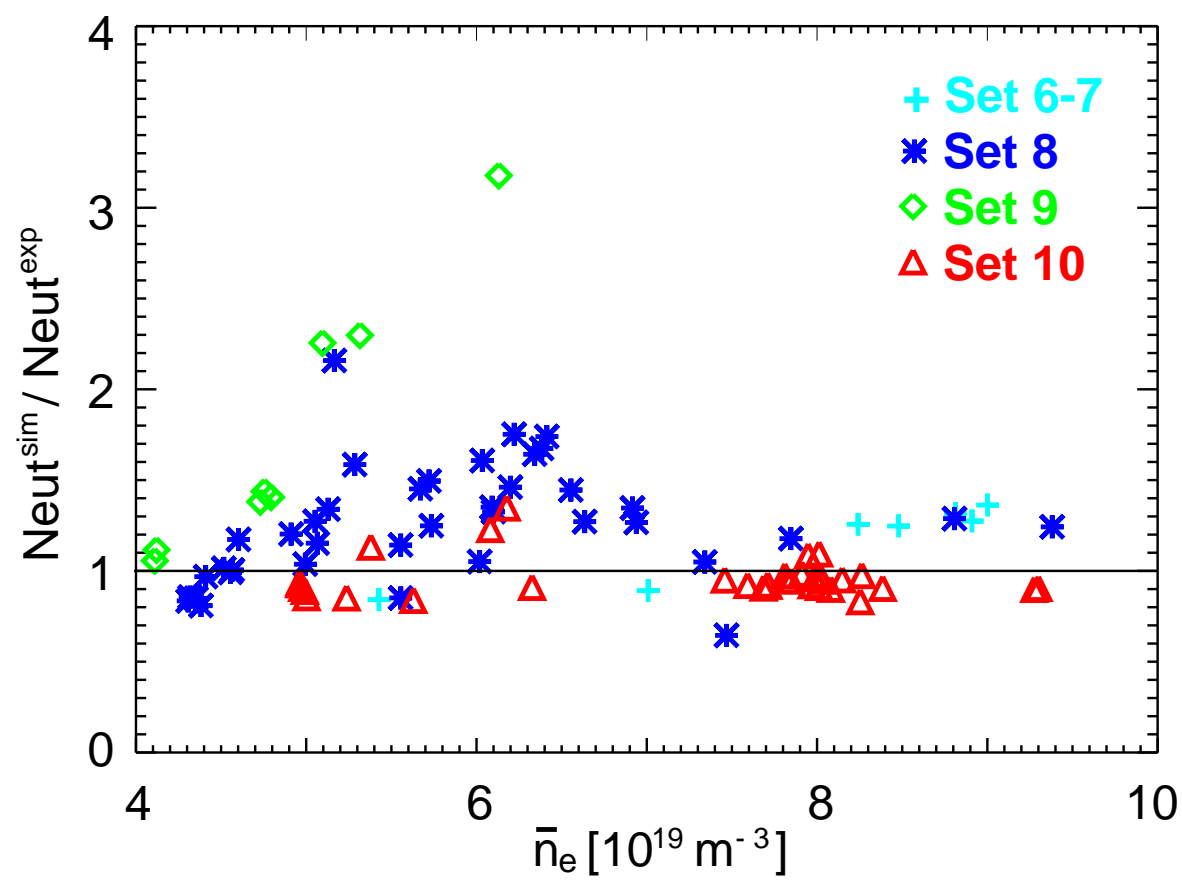

Figure 6. Ratio between modelled and experimental neutron rate versus $\bar{n}_{e}$, assuming $\mathrm{Z}_{\text {eff }}$ from the empirical formula. Colors and symbols like in Fig. 1.

correction factor, and the correlation coefficients with the linear fit are summarised in Table 2. For set 8 some systematic factor remains even with the addition of the density dependent formula for $\mathrm{Z}_{\text {eff }}$, despite the significant improvement from 2.13 to 1.45 . This factor must be considered to normalise the data of a given calibration period, in order to compare mesurements from different calibration phases, as described in Section 7. However, the scattering of the points is low, as documented by Fig. 6 and by the correlation factor 0.93 in Table 2. Therefore, the modelling appears to be accurate and 


\begin{tabular}{|c|c|c|c|c|}
\hline Set & Fit coeff. & Fit coeff. $\left(\mathrm{Z}_{e f f}\right)$ & Correlation & Correlation $\left(\mathrm{Z}_{e f f}\right)$ \\
\hline 6 & 1.49 & $\mathbf{1 . 3 0}$ & 0.99 & 0.99 \\
7 & 1.51 & $\mathbf{0 . 8 5}$ & 1.0 & 1.0 \\
8 & 2.13 & $\mathbf{1 . 4 5}$ & 0.90 & $\mathbf{0 . 9 3}$ \\
9 & 3.23 & $\mathbf{2 . 0}$ & 0.73 & 0.59 \\
10 & 1.19 & $\mathbf{0 . 9 3}$ & 0.91 & $\mathbf{0 . 9 7}$ \\
\hline
\end{tabular}

Table 2. Table of the fit coefficient for the linear regression of the simulated neutron rate as a function of the measured one, as well as their correlation factors. Without and with the $\mathrm{Z}_{\text {eff }}$ formula. Most coefficients improve using the $\mathrm{Z}_{\text {eff }}$ formula (bold font).

it can be used as a verification a posteriori of the calibration for that phase.

Set 9 has, instead, also a high scattering and a clear trend with density, as Fig. 6 shows. It has to be noted, however, that this set has only 9 points and therefore a poor statistics. Moreover, the trend with density is opposite to the one shown in Fig. 2 for the large sets 8 and 10, i.e. for the vast majority of the analysed discharges, where the mismatch is larger for low density. A diffusion coefficient of fast ions would not help either, because it reduces the neutron rate of low density discharges more than the high density cases, as shown in Fig. 3. The discrepancy is observed in only 3 time intervals in Fig. 6. For two of them (\#19992 and \#20008) it is associated with high MHD activity, which can reduce the fast ion content, while unaccurate measurement of the kinetic profiles could also play a role. 
The relative contribution of thermonuclear, beam-plasma and beam-beam neutrons is summarised in Fig. 7. The beam-plasma component is indeed dominant, as expected,

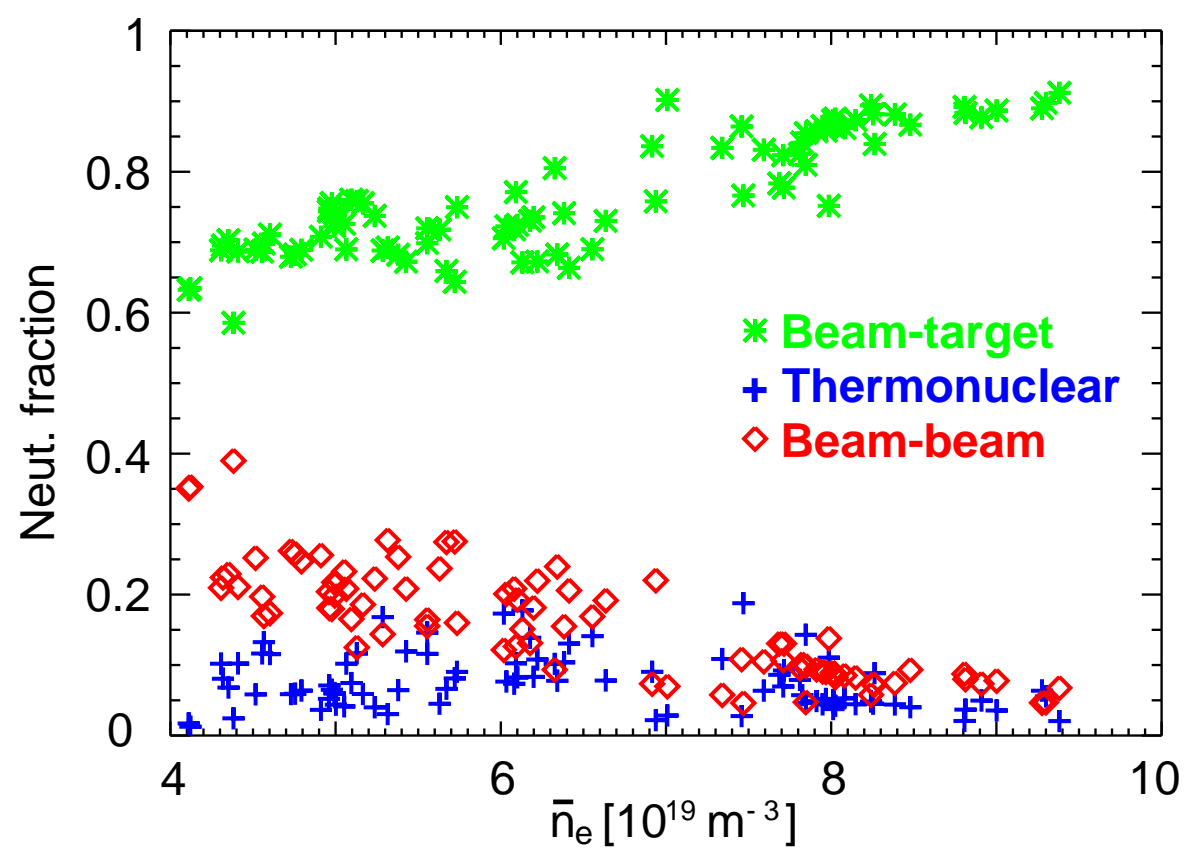

Figure 7. Modelled relative contribution to the neutron rate.

although the thermonuclear and beam-beam components cannot be neglected.

\subsection{Impact of the profile measurement quality}

The dataset 10, corresponding to the most recent calibration phase, exhibits by far the smallest scattering between the predicted and the measured neutron rate. The only diagnostics with a significant change over this time period is the IDA evaluation of the $n_{e}$ profiles, not routinely available for sets 1-9. Further evidence of the better consistency of IDA $n_{e}$ profiles is presented in Fig. 8, including both IDA profiles and directly Abel-inverted interferometry measurements. Retaining only the IDA points, the correlation coefficient with the linear fit increases from 0.97 to 0.99 , for a subset of 


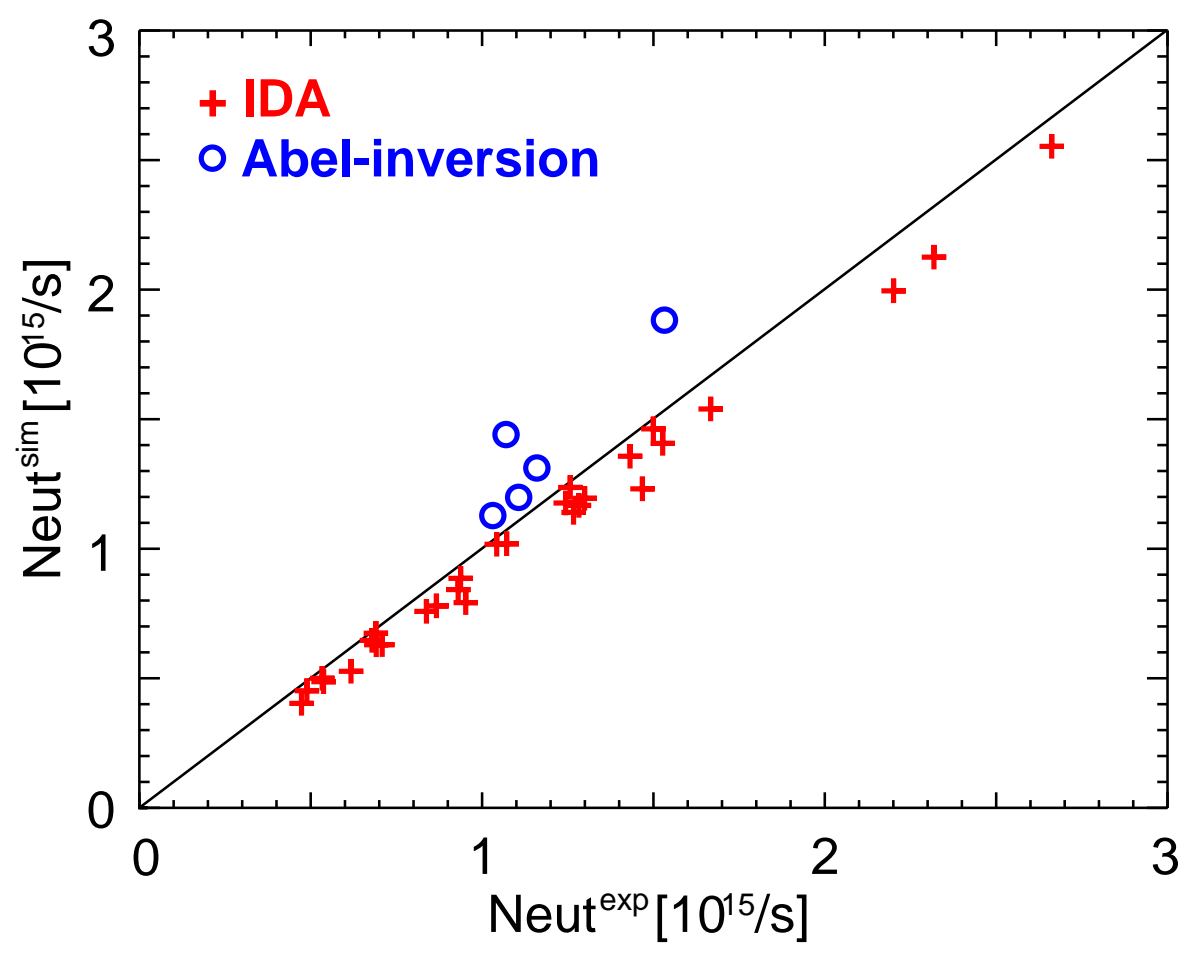

Figure 8. Predicted versus measured neutron rate. Simulations with IDA $n_{e}$ profiles (red crosses) are in excellent agreement with the measured neutron rate, as compared to Abel-inverted interferometry measurements (blue circles).

34 plasma discharges. For a detailed comparison between modelled and experimental neutron rates, an accurate measurement of $n_{e}$ is necessary.

\section{Dependence study}

The dependence of the neutron rate on the plasma energy $W_{M H D}$ has been investigated in [26]. With the present database we want to determine such a dependence. First of all, in order to compare sets of different calibration avoiding systematic errors we have divided each dataset by the respective fit coefficients, summarised in Table 2 . In Fig. 9 the corrected experimental neutron rate is plotted against $W_{M H D}$. While in 


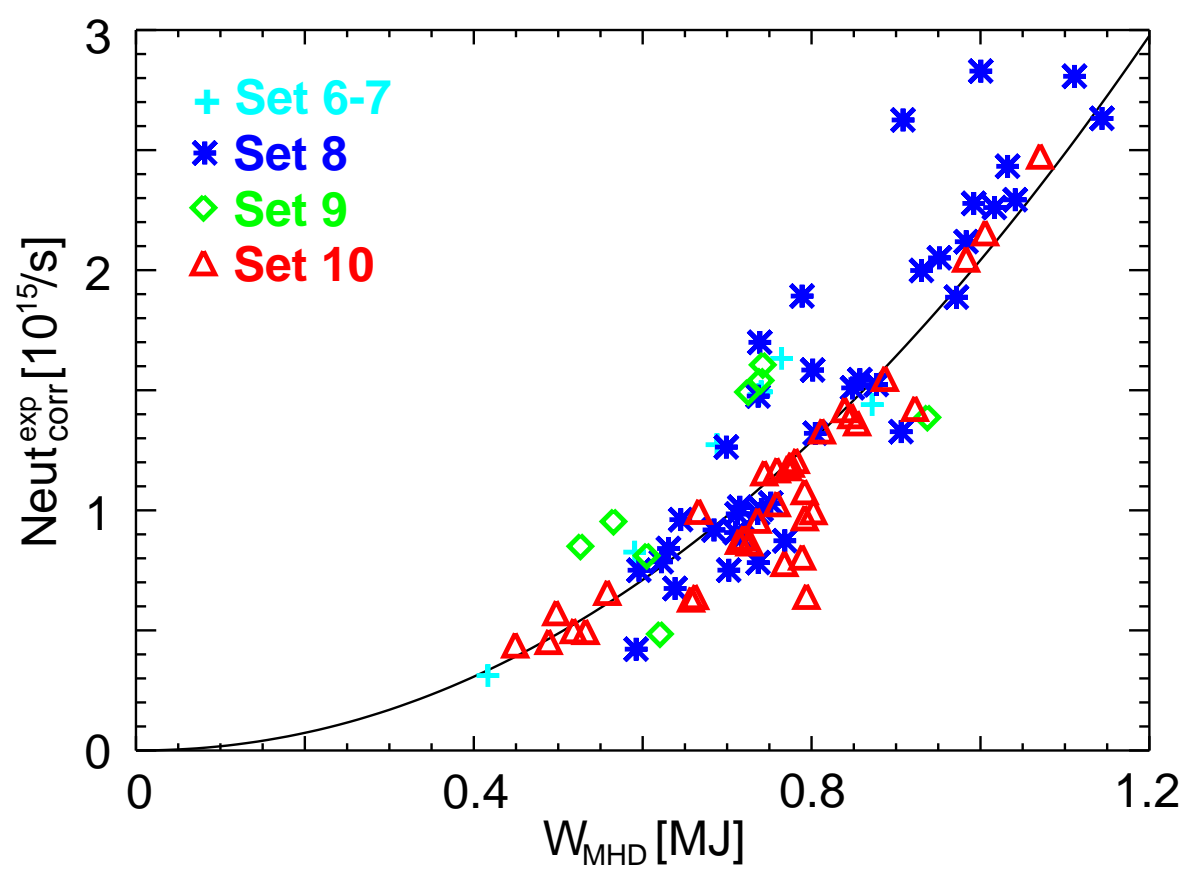

Figure 9. Measured neutron rate with calibration correction as a function of the plasma stored energy. Colors and symbols like in Fig. 1. The black curve represents the best fit from a power regression.

Reference [26] the parabolic behaviour was found to be only an upper limit, Fig. 9 shows a monotonic trend despite some scattering. This significant improvement in the statistical trend is due to the availability of the TRANSP simulations, which determine the calibration factors and therefore allow to superpose data from different calibration phases.

A single-dimensional power regression analysis delivers a scaling of the neutron rate which is, in fact, quadratic in $W_{M H D}$, as expected:

$$
S=809 W_{M H D}^{2.067}
$$


taking $\mathrm{W}_{M H D}$ in $\mathrm{J}$ and the neutron rate in $\mathrm{s}^{-1}$. The curve corresponding to the fit is overplotted in Fig. 9 (black curve). However, it is more significant to find a fit with respect to engineering parameters, which are a proper input of a plasma discharge, whereas physical parameters like $W_{M H D}$ are not known a priori. Additionally we include in the regression the confinement improvement factor $H_{I P B 98(y, 2)}$, which describes the deviation of the confinement from the regression derived in [14] and cannot, therefore, be hidden in any set of engineering parameters. According to Table $3 P_{N B I}$ is well correlated with $W_{M H D}$, as expected; it is certainly a significant parameter for the neutron rate, considering the dominance of beam-plasma reactions to the total neutron production rate shown in Fig. 7. For a given $P_{N B I}$ also the density and the electron temperature are expected to play a role. While the density can be considered an engineering parameter, the temperature at a given input power and plasma density has a good proxy in the confinement improvement factor. In fact, after some preliminary single parameter regressions, the most significant fitting parameters for the measured neutron rate turn out to be $P_{N B I}, H_{I P B 98(y, 2)}, I_{p l}$ and $\bar{n}_{e}$. The ranges of these parameters are summarised in Table 1. Their cross-correlations are shown in Table 3. There is a non-negligible cross-correlation between $\bar{n}_{e}$ and $P_{N B I}$, as high NBI power is applied usually to high density discharges. As a consequence, the best fit will not be perfectly unique, as these parameters can "trade" their exponents to some extent, still delivering a good fit. The best regression is found to be

$$
S \propto P_{N B I}^{1.39} H_{I P B 98(y, 2)}^{1.66} I_{p l}^{1.57} \bar{n}_{e}^{-0.58}
$$




\begin{tabular}{|c|ccccc|}
\hline & $\mathrm{S}$ & $P_{N B I}$ & $H_{I P B 98(y, 2)}$ & $I_{p l}$ & $\bar{n}_{e}$ \\
\hline $\mathrm{S}$ & 1 & $\mathbf{0 . 6 3}$ & 0.40 & 0.24 & -0.0097 \\
$P_{N B I}$ & & 1 & -0.14 & -0.019 & $\mathbf{0 . 5 2}$ \\
$H_{I P B 98(y, 2)}$ & & & 1 & -0.075 & -0.26 \\
$I_{p l}$ & & & & 1 & -0.071 \\
$\bar{n}_{e}$ & & & & & \\
\hline
\end{tabular}

Table 3. Cross-correlation of the regression parameters. Strong correlations are highlighted in bold.

Such a regression can be understood in terms of physics dependences and it is well consistent with beam-plasma reactions being the dominant fraction of the total neutron rate. For beam-plasma neutrons it is

$$
S \propto T^{3 / 2} P_{N B I} \propto\left(\frac{W}{\bar{n}_{e}}\right)^{3 / 2} P_{N B I}
$$

Taking the common IPB98 $(y, 2)$ scaling from the multi-machine database in Reference [14], in terms of our parameters it is $\tau_{E}=H_{I P B 98(y, 2)} \tau_{I P B 98(y, 2)} \propto$ $H_{I P B 98(y, 2)} I_{p l}^{0.93} P_{\text {loss }}^{-0.69} \bar{n}_{e}^{0.41}$. We can assume $P_{N B I}$ to be the most significant heating source in our plasmas, because we have selected the discharge database excluding ICRH and ECRH phases; moreover, we selected only discharges with $P_{N B I}>5 \mathrm{MW}$, significantly larger than the ohmic power in ASDEX Upgrade. As a consequence, in stationary conditions we can assume $P_{\text {loss }} \approx P_{N B I}$ and $W \approx P_{N B I} \tau_{E}$. Substituting in Equation 6 yields

$$
S \propto P_{N B I}^{1.47} H_{I P B 98(y, 2)}^{1.5} I_{p l}^{1.40} \bar{n}_{e}^{-0.89}
$$


which is in excellent agreement with the experimental power regression in Equation 5. This provides experimental evidence of the theoretical finding that in ASDEX Upgrade NBI-heated H-mode plasmas beam-target reactions are the dominant contribution to the neutron production. An independent assessment will be possible with neutron spectroscopy [17], as soon as the characterisation of the detector will be complete, thus allowing the unfolding of the neutron energy spectra.

\section{Conclusions}

In this paper an extensive validation of the theoretical prediction of the neutron rate production is carried out, based on a comprehensive database of ASDEX Upgrade NBI heated H-mode discharges, including some improved H-mode plasmas. The modelling highlights the importance of the accurate measurement of the density profiles, showing a considerable improvement since the Integrated Data Analysis has been introduced. Moreover, the simulations help identifying possible systematic errors due to uncertainties in the calibration procedure, which is shown to be accurate up to roughly $40 \%$ over the years. The modelling procedure applied in this paper is shown to be an efficient calibration quality check for new calibrations of neutron detectors in tokamaks.

On the other hand, the experimental results show that a good choice of the $\mathrm{Z}_{\text {eff }}$ value is needed for the simulation to deliver accurate predictions. It is also shown that no anomalous fast ion transport is necessary to improve the prediction capability of the TRANSP simulations, because only unrealistic high values of $D_{f i} \approx 2 \mathrm{~m}^{2} / \mathrm{s}$ would have 
a significant impact; an empirical formula for $\mathrm{Z}_{\text {eff }}$ derived in Reference [11] is actually good enough to considerably reduce the scattering between experiment and theory and to suppress the systematic trend which overpredicts the neutron rate at low density.

Both the theoretical and the experimental evidence highlight the dominant role of the beam-plasma neutrons in NBI heated discharges with $P_{N B I}>5 M W$ : TRANSP returns the different contributions as separate output, while a simple 0D derivation of the key dependences of the beam-plasma neutron rate shows a remarkable agreement with the power regression of the experimental database.

[1] J. Wesson, D. J. Campbell, "Tokamaks", Oxford University Press, USA, 2004

[2] S. Günter et al , Nuclear Fusion 47 (2007) 920

[3] B. Geiger et al , Plasma Phys. Controlled Fusion 53 (2011) 065010

[4] E. Ruskov et al , Nuclear Fusion 35 (1995) 1099

[5] R. V. Budny et al, "Transport in JET H-mode plasmas with beam and ion cyclotron heating", 39th EPS Conference on Plasma Physics, Stockholm 2012, P1.052, http://ocs.ciemat.es/epsicpp2012pap/pdf/P1.052.pdf

[6] B. Wolle et al , Plasma Phys. Controlled Fusion 36 (1994) 1051

[7] I. Voitsekhovitch et al, Nuclear Fusion 47 (2007) 599

[8] I. Wodniak et al , TRANSP modelling of neutron emissivity on MAST, 39th EPS Conference on Plasma Physics, Stockholm 2012, P5.077, http://ocs.ciemat.es/epsicpp2012pap/pdf/P5.077.pdf

[9] R. Bilato et al , Nuclear Fusion 51 (2011) 103034

[10] A. Pankin, D. McCune, R. Andre et al , Comp. Phys. Comm. 159, No. 3 (2004) 157

[11] H. Meister et al, $\mathrm{Z}_{\text {eff }}$ Behaviour in ASDEX Upgrade H-mode Discharges, 30th EPS Conference on Plasma Physics and Controlled Fusion, St. Petersburg 2003, P1.136, 
http://epsppd.epfl.ch/StPetersburg/PDF/P1_136.PDF

[12] R. Neu et al, Plasma Phys. Controlled Fusion 49 (2007) B59

[13] R. Fischer et al , Plasma Phys. Controlled Fusion 45 (2003) 1095

[14] ITER Physics Basis Expert Group on Energetic Particles, Heating and Current Drive, ITER Physics Basis Editors: Chapter 6: Plasma auxiliary heating and Current drive, Nucl. Fusion 39 (1999) 2495

[15] C. Höhbauer, IPP report IPP 1/339 (2010)

[16] L. Giacomelli et al , Review of Scientific Instruments 82 (2011) 123504

[17] G. Tardini et al , Journal of Instrumentation 7 (2012) C03004

[18] J. D. Strachan et al , Nuclear Fusion 33 (1993) 991

[19] H. - S. Bosch, G. M. Hale, Nuclear Fusion 32 (1992) 611

[20] R. K. Janev et al , Nuclear Fusion 29 (1989) 2125

[21] M. Kraus et al , IPP report IPP 5/122 (2010)

[22] W. W. Heidbrink et al, Physical Review Letters 103 (2009) 175001

[23] T. Hauff et al , Physical Review Letters 102 (2009) 075004

[24] W. W. Heidbrink et al , Nuclear Fusion 42 (2002) 972

[25] H. W. Hendel et al , Journal of Fusion Energy 5(3) (1986) 231

[26] H, Zohm et al , 29th EPS Conference on Plasma Physics and Controlled Fusion, Montreaux 2002, P1.043 